\title{
EXISTENCE OF MULTIPLE PERIODIC SOLUTIONS FOR A SEMILINEAR EVOLUTION EQUATION
}

\author{
NORIMICHI HIRANO
}

(Communicated by Walter D. Littman)

ABSTRACT. In this paper, we consider the existence of multiple periodic solutions for the problem

$$
\begin{gathered}
\frac{d u}{d t}+L u=g(u)+h, \quad t>0, \\
u(0)=u(T),
\end{gathered}
$$

where $L$ is a uniformly strongly elliptic operator with domain $D(L)=H_{0}^{m}(\Omega)$, $g: R \rightarrow R$ is a continuous mapping, $T>0$ and $h:(0, T) \rightarrow H_{0}^{m}(\Omega)$ is a measurable function.

\section{INTRODUCTION}

Let $\Omega \subset R^{n}$ be a bounded domain with a smooth boundary $\partial \Omega$ and

$$
L:=\sum_{0 \leq|\alpha|,|\beta| \leq m}(-1)^{|\beta|} D^{\beta}\left(a_{\alpha \beta}(x) D^{\alpha}\right)
$$

a differential operator of order $2 m$ with domain $D(L)=H_{0}^{m}(\Omega)$ satisfying the following conditions.

(L1) $L$ is uniformly strongly elliptic, that is, there exists $C>0$ such that

$$
\sum_{|\alpha|=|\beta|=m} a_{\alpha \beta}(\chi) \xi^{\alpha} \xi^{\beta} \geq C|\xi|^{2 m} \quad \text { for all } \xi \in R^{n} \text { and } x \in \Omega \text {. }
$$

(L2) $a_{\alpha \beta}=a_{\alpha \beta}$ are real valued functions in $C^{\infty}(\Omega)$.

Our purpose in the present paper is to consider the existence of multiple solutions for the problem of the form

$$
\begin{gathered}
\frac{d u}{d t}+L u=g(u)+h(t), \quad t>0, \\
u(0)=u(T),
\end{gathered}
$$

where $T>0, h:(0, \infty) \rightarrow L^{2}(\Omega)$ is a $T$-periodic function and, $g: R \rightarrow R$ is a continuous function with $g(0)=0$.

Received by the editors August 1, 1988.

1980 Mathematics Subject Classification (1985 Revision). Primary 35B10, 35F25.

Key words and phrases. Periodic solution, elliptic operator, evolution equation. 
The existence of periodic solutions for problems of this kind has been studied by many authors (see Nieto [8] which also contains many references). But few seem to be known about the multiplicity of the periodic solutions. In [3], Amann established a multiplicity result for the problem (P) for the case in which $L$ is second order. The method employed in [1] is based on the super-subsolution method. Our argument in the presenet paper is based on the Leray-Schauder fixed point theorem.

To state our result, we need some notations. Throughout the rest of this paper, we assume that $L$ satisfies the condition (L1) and (L2). Let $\lambda_{1}<\lambda_{2}<$ $\cdots<\lambda_{k}<\cdots$ denote the eigenvalues of the selfadjoint realization in $L^{2}(\Omega)$ of $L$. If $X$ is a Banach space, we denote by $L^{p}(0, T ; X) \quad(1 \leq p \leq \infty)$ the space of all $X$-valued strongly measurable functions such that $\int_{0}^{T}\|u(t)\|^{p} d t<$ $\infty$. $W^{1, p}(0, T ; X)$ denotes the space of functions $u \in L^{p}(0, T ; X)$ such that $d u / d t \in L^{p}(0, T ; X)$, where $d u / d t$ is the derivative in the sense of distribution (see [4]). We denote by $|\cdot|$ the norm of $L^{2}(\Omega)$.

We suppose that $g$ satisfies the following condition

$$
-\infty<a_{*} \leq a^{*}<\lambda_{1}<b_{*} \leq b^{*}<\lambda_{2},
$$

where

$$
a_{*}=\inf _{s \neq t} \frac{g(t)-g(s)}{t-s}, \quad a^{*}=\limsup _{|t| \rightarrow \infty} \frac{g(t)}{t}, \quad b_{*}=\liminf _{|t| \rightarrow \infty} \frac{g(t)}{t},
$$

and $b^{*}=\sup _{s \neq t} \frac{g(t)-g(s)}{t-s}$.

Theorem. Suppose that $g$ satisfies the condition $(\mathrm{G})$. Then there exists $M>0$ such that for each $T$-periodic function $h \in W^{1, \infty}\left(0, T ; L^{2}(\Omega)\right)$ with

$$
\sup \{|h(t)|: t \in(0, T)\} \leq M,
$$

the problem $(\mathrm{P})$ possesses at least two solutions in $W^{1, \infty}\left(0, T ; L^{2}(\Omega)\right)$.

Remark. In (G), we have implicitly supposed $\lambda_{1}$ is single.

\section{Preliminaries}

In the following, we write $H, V$ and $V^{*}$ instead of $L^{2}(\Omega), H_{0}^{m}(\Omega)$ and $H^{-m}(\Omega)$, respectively. The pairing between $H_{0}^{m}(\Omega)$ and $H^{-m}(\Omega)$ is denoted by $\langle\cdot, \cdot\rangle$. If $u, v \in L^{2}(\Omega)$, then $\langle u, v\rangle$ is the ordinary inner product of $L^{2}(\Omega)$. Here we observe that we may assume without any loss of generality that $\lambda_{1}>0$. In fact, the problem (P) is equivalent to $d u / d t+L_{1} u=g_{1}(u)+h$, $u(0)=u(T)$, where $L_{1} u=L u+\lambda u$ and $g_{1}(u)=\lambda u+g(u)$. If $\lambda+\lambda_{1}>0$, the first eigenvalue of $L_{1}$ is positive, and $g_{1}$ satisfies the condition $(G)$ with $\lambda_{1}, \lambda_{2}$ replaced by $\lambda+\lambda_{1}, \lambda+\lambda_{2}$, respectively. Also we may assume that $0<a_{*}$. We denote by $\|\cdot\|$ the norm of $H_{0}^{m}(\Omega)$ defined by $\|u\|^{2}=\langle L u, u\rangle$ for $u \in H_{0}^{m}(\Omega)$. From the observation above, the norm $\|\cdot\|$ is equivalent to the Sobolov norm on $H_{0}^{m}(\Omega)$. The norm of $H^{-m}(\Omega)$ is denoted by $\|\cdot\|_{*}$. Let $V_{1}$ 
and $V_{2}$ be the subspaces of $H_{0}^{m}(\Omega)$ spanned by the eigenspaces corresponding to the eigenvalues $\left\{\lambda_{2}, \lambda_{3}, \ldots\right\}$ and $\left\{\lambda_{1}\right\}$, respectively. Then $V_{1}$ and $V_{2}$ are orthogonal in $L^{2}(\Omega)$. Let $\phi$ be a normalized eigenfunction corresponding to $\lambda_{1}$. Then $\phi \in L^{\infty}(\Omega)$ and $V_{2}=\{k \phi: k \in R\}$. We denote by $P_{1}$ and $P_{2}$ the projections from $L^{2}$ onto $V_{1}$ and $V_{2}$, respectively. We denote by $|\Omega|$ the measure of $\Omega$. Suppose that $g$ satisfies the condition $(G)$. Then there exist positive constants $\alpha, \beta, \rho$, and $\delta$ such that

$$
\alpha<\lambda_{1}, \quad g(t) / t \leq \alpha \quad \text { for all } t \text { with }|t| \geq \delta .
$$

and

$$
\beta>\lambda_{1}, \quad g(t) / t \geq \beta \quad \text { for all } t \text { with } 0<|t| \leq p .
$$

Lemma 1. There exists $\omega_{1}>0$ such that

$$
\langle L u-g(u), u\rangle \geq \omega_{1}\|u\|^{2}-\delta \cdot \max \{g(t):|t| \leq \delta\}|\Omega|
$$

for all $u \in V$.

Proof. From (2.1), we have that

$$
\begin{aligned}
\langle L u-g(u), u\rangle & \geq\|u\|^{2}-\alpha|u|^{2}-\int_{u<\delta} a(u) u d x \\
& \geq\|u\|^{2}-\alpha|u|^{2}-\delta \cdot \max \{g(t):|t| \leq \delta\}|\Omega|
\end{aligned}
$$

for each $u \in V$. Then since $\alpha<\lambda_{1}$, the inequality (2.3) follows:

Lemma 2. There exist $c_{0}>0$ and $\omega_{2}>0$ such that

$$
\left\langle L u-g(u), u-2 P_{2} u\right\rangle \geq \omega_{2}\|u\|^{2} \quad \text { for all } u \in V \text { with }\left\|P_{2} u\right\| \leq c_{0} .
$$

Proof. Let $b$ be a positive constant with $b^{*}<b<\lambda_{2}$. We choose $d>0$ so small that

$$
\left(b-b^{*}\right) p^{2}-\left(b-a_{*}\right) d^{2}>0 \text { for all } p \text { with } p \geq \rho-d .
$$

We next choose $c_{0}>0$ such that $\sup _{x \in \Omega}|w(x)| \leq d$ for all $w \in V_{2}$ with $\|w\| \leq c_{0}$. In fact, we can choose such $c_{0}>0$ because $\phi \in L^{\infty}(\Omega)$. Let $u \in V$ such that $\left\|P_{2} u\right\| \leq c$. For simplicity, we put $v=P_{1} u$ and $w=P_{2} u$. Also we set $A=\{x \in \Omega:|u(x)|>\rho\}$. Then we have

$$
\left\langle L u-g(u), u-2 P_{2} u\right\rangle \geq\|v\|^{2}-\lambda_{1}|w|^{2}-\int_{\Omega} g(u)(v-w) d x .
$$

Let $x \in A$. If $|w(x)|>|v(x)|$, then noting that $u(x)(v-w)(x)<0$ we find by $(\mathrm{G})$ that

$$
\begin{aligned}
-g(u(x))(v-w)(x) & \geq a_{*}|w(x)|^{2}-a_{*}|v(x)|^{2} \\
& \geq a_{*}|w(x)|^{2}-b^{*}|v(x)|^{2} .
\end{aligned}
$$

If $|w(x)| \leq|v(x)|$, then we have again by $(\mathrm{G})$ that

$$
-g(u(x))(v-w)(x) \geq a_{*}|w(x)|^{2}-b^{*}|v(x)|^{2} .
$$


Then from (2.5) and the inequalities above, we have that

$$
\begin{aligned}
& -\lambda_{1}|w(x)|^{2}-g(u(x))(v-w)(x) \geq\left(b-\lambda_{1}\right)|w(x)|^{2}-b|v(x)|^{2} \\
& \quad+\left\{\left(b-b^{*}\right)|v(x)|^{2}-\left(b-a_{*}\right)|w(x)|^{2}\right\} \\
& \geq\left(b-\lambda_{1}\right)|w(x)|^{2}-b|v(x)|^{2} .
\end{aligned}
$$

Let $x \in \Omega \backslash A$. Then we have by (2.2) and (G)

$$
-g(u(x))(v-w)(x) \geq \beta|w(x)|^{2}-b^{*}|v(x)|^{2} .
$$

Then we find that

$$
-\lambda_{1}|w(x)|^{2}-g(u(x))(v-w)(x) \geq\left(\beta-\lambda_{1}\right)|w(x)|^{2}-b^{*}|v(x)|^{2} .
$$

Then combining (2.7), (2.9) with (2.6), we obtain that

$$
\begin{aligned}
\left\langle L u-g(u), u-2 P_{2} u\right\rangle & \geq\|v\|^{2}-\lambda_{1}|w|^{2}-\int_{\Omega} g(u)(v-w) d x, \\
& \geq\|v\|^{2}-b|v|^{2}+\left(\beta-\lambda_{1}\right)|w|^{2} .
\end{aligned}
$$

Since $b<\lambda_{2}$ and $\left(\beta-\lambda_{1}\right)>0$, we can choose $\omega_{2}>0$ such that

$$
\|v\|^{2}-b|v|^{2}+\left(\beta-\lambda_{1}\right)|w|^{2} \geq \omega_{2}\|u\|^{2}
$$

and this completes the proof.

We now define a functional $\Phi: V \rightarrow R$ by

$$
\Phi(v)=\frac{1}{2}\langle L v, v\rangle-\int_{\Omega} \int_{0}^{v(x)} g(\tau) d \tau d x \quad \text { for each } v \in V .
$$

It is easy to see that $\left\langle\Phi^{\prime}(v), w\right\rangle=\langle L v-g(v), w\rangle$ for $v, w \in V$. It is also easy to verify that for each $w \in V_{2}$, the mapping $\Phi(\cdot+w): V_{1} \rightarrow R$ is strictly convex. In fact, we have from $(G)$ that

$$
\begin{aligned}
\left\langle\Phi^{\prime}\left(v_{1}+w\right)-\Phi^{\prime}\left(v_{2}+w\right), v_{1}-v_{2}\right\rangle & \geq\left\|v_{1}-v_{2}\right\|^{2}-b^{*}\left|v_{1}-v_{2}\right|^{2} \\
& \geq \omega_{2}\left\|v_{1}-v_{2}\right\|^{2}
\end{aligned}
$$

for each $w \in V_{2}$ and each $v_{1}, v_{2} \in V_{1}$. This implies that $\Phi(\cdot+w)$ is strictly convex. Then we have that for each $w \in V_{1}$, there exists a unique element $v_{0} \in V_{1}$ such that

$$
\Phi\left(v_{0}+w\right)=\min \left\{\Phi(v+w): v \in V_{1}\right\} .
$$

Here we set $\theta(w)=v_{0}$. Then $\theta: V_{2} \rightarrow V_{1}$ is a continuous mapping satisfying that

$$
\langle L(\theta(w)+w)=g(\theta(w)+w), z\rangle=0 \quad \text { for all } z \in V_{1} .
$$

Let $w \in V_{2}$. Then it also follows from the strict convexity of $\Phi(\cdot+w)$ that

$$
\begin{aligned}
& v=\theta(w) \text { if and only if } \\
& \langle L(v+w)-g(v+w), z\rangle=0 \quad \text { for all } z \in V_{1} .
\end{aligned}
$$


We next define a continuous function $F: R \rightarrow R$ by

$$
F(s)=\Phi(\theta(s \phi)+s \phi) \quad \text { for } s \in R .
$$

Then it is not difficult to see that

$$
F^{\prime}(x)=\langle L(\theta(s \phi)+s \phi)-g(\theta(s \phi)+s \phi), \phi\rangle,
$$

(See Theorem 2.3 of [2].) It also follows from (2.13) and (2.15) that if $F(s)=$ $\min \{F(\tau): \tau \in R\}$, then

$$
L(\theta(s \phi)+s \phi)-g(\theta(s \phi)+s \phi)=0 .
$$

Lemma 3. (1) There exists $r>0$ such that $F(s) \geq 0$ for all $s$ with $|s| \geq r$;

$$
\min \{F(s): s \in(0, r)\}<0 \text { and } \min \{F(s): s \in(-r, 0)\}<0 \text {. }
$$

Proof. We have by (2.13) and Lemma 1 that

$$
\begin{aligned}
F^{\prime}(s) s & =\langle L(\theta(s \phi)+s \phi)-g(\theta(s \phi)+s \phi), s \phi\rangle \\
& =\langle L(\theta(s \phi)+s \phi)-g(\theta(s \phi)+s \phi), \theta(s \phi)+s \phi\rangle \\
& \geq \omega_{1}\|\theta(s \phi)+s \phi\|^{2}-\delta \cdot \max \{g(\tau):|\tau| \leq \delta\}|\Omega| \\
& \geq s^{2} \omega_{1}\|\phi\|-\delta \cdot \max \{g(\tau):|\tau| \leq \delta\}|\Omega| .
\end{aligned}
$$

Then from the inequality above, we can choose $r>0$ such that $F(s) \geq 0$ for all $s$ with $|s| \geq r$. We next show that (2) holds. From (2.13) and Lemma 2, we have that

$$
F^{\prime}(s) s=\langle L(\theta(s \phi)+s \phi)-g(\theta(s \phi)+s \phi), s \phi\rangle<0
$$

for all $s$ with $0<|s| \leq c_{0}$. Then since $F(0)=0$, (2) follows from (1) and the inequality above.

\section{Proof of THEOREM}

The Proof of Theorem will be accomplished by a series of lemmas. From Lemma 3, we can see that there exist constants $\alpha_{ \pm}, \beta_{ \pm}, \alpha_{ \pm}^{*}$, and $\beta_{ \pm}^{*}$ such that

$$
\begin{gathered}
\alpha_{-}^{*}<\alpha_{-} \leq \beta_{-}<\beta_{-}^{*}<0<\alpha_{+}^{*}<\alpha_{+} \leq \beta_{+}<\beta_{+}^{*}, \\
F(s)=m_{+}=\min \{F(\tau): \tau \in(0, r)\} \quad \text { on }\left(\alpha_{+}, \beta_{+}\right), \\
F(s)=m_{-}=\min \{F(\tau): \tau \in(-r, 0)\} \quad \text { on }\left(\alpha_{-}, \beta_{-}\right) \text {and } \\
\left|F^{\prime}(s)\right|>0 \quad \text { for all } s \in\left(\alpha_{ \pm}^{*}, \beta_{ \pm}^{*}\right) \backslash\left(\alpha_{ \pm}, \beta_{ \pm}\right) .
\end{gathered}
$$

For simplicity, we will assume that $m_{-}=m_{+}=m$. Here we choose a negative number $c$ such that $m<c<\min \left\{F\left(\alpha_{ \pm}^{*}\right), F\left(\beta_{ \pm}^{*}\right)\right\}$. Then from the definition above, $V_{ \pm}=\{\tau \in R: F(\tau) \leq c\} \cap\left(\alpha_{ \pm}^{*}, \beta_{ \pm}^{*}\right)$ are closed intervals which contain $\left(\alpha_{+}, \beta_{+}\right)$and $\left(\alpha_{-}, \beta_{-}\right)$, respectively. We set

$$
K_{ \pm}=\{v \in V: \Phi(v) \leq c\} \cap\left\{v \in V: P_{2} v \in V_{ \pm}\right\} \quad \text { and } \quad \widetilde{K}_{ \pm}=\overline{c o} K_{ \pm},
$$

where $\overline{\operatorname{co}} A$ denotes the closed convex hull of a subset $A$ of $V$. Then from (2.12), we can see that $K_{ \pm}$are bounded closed subsets of $V$. Since $V$ is compactly embedded in $H, K_{ \pm}$is compact in $H$. We also have by the definition that $\widetilde{K}_{ \pm} \subset\left\{v \in V: P_{2} v \in V_{ \pm}\right\}$. 
Lemma 4. There exist mappings $Q_{ \pm}: \widetilde{K}_{ \pm} \rightarrow K_{ \pm}$such that $Q_{ \pm}$are continuous with respect to the norm of $H$ and $Q_{ \pm} x=x$ for each $x \in K_{ \pm}$.

Proof. Let $w \subset V_{+}$. Then since the functional $\Phi(\cdot+w)$ is convex, we have that $P_{2}^{-1}(w) \cap K_{+}$is compact convex in $H$. Here we denote by $Q_{w}$ the metric projection from $H$ onto $P_{2}^{-1}(w) \cap K_{+}$. Then $Q_{w}$ is continuous with respect to the norm of $H$. We define a mapping $Q_{+}$from $\widetilde{K}_{+}$onto $K_{+}$by $Q_{+} x=Q_{P_{2} x} x$ for each $x \in \widetilde{K}_{+}$. Then it is easy to verify that the mapping $Q_{+}$is continuous on $\widetilde{K}_{+}$and that $Q_{+} x=x$ for $x \in K_{+}$. By the same argument, we can find a continuous mapping $Q_{-}$satisfying the required property.

We next choose open sets $U_{ \pm} \subset V$ such that

$$
\left\{\theta(t \phi)+t \phi: t \in\left(\alpha_{ \pm}, \beta_{ \pm}\right)\right\} \subset U_{ \pm} \subset K_{ \pm} \quad \text { and } \quad \sup \left\{\Phi(v): v \in U_{ \pm}\right\}<c .
$$

Then we have the following lemma:

Lemma 5. There exist $d_{ \pm}>0$ such that

$$
\|L v-g(v)\|_{*} \geq d_{ \pm} \quad \text { for all } v \in K_{ \pm} \backslash U_{ \pm} .
$$

Proof. Suppose that there exists a sequence $\left\{v_{n}\right\} \subset K_{+} \backslash U_{+}$such that $\left\|L v_{n}-g\left(v_{n}\right)\right\|_{*} \rightarrow 0$, as $n \rightarrow \infty$. Since $K_{+}$is a bounded subset of $V$, we may assume that $v_{n} \rightarrow v \in K_{+}$weakly in $V$. Then from the compactness of $K_{+}$in $L^{2}$, we have that $v_{n} \rightarrow v$ strongly in $L^{2}$. Thus we find that $L v-g(v)=0$. Then from (*), it follows that $v=\theta\left(\left\|P_{2} v\right\| \phi\right)+\left\|P_{2} v\right\| \phi$. Here we suppose that $\left\|P_{2} v\right\| \in\left(\alpha_{+}^{*}, \beta_{+}^{*}\right) \backslash\left(\alpha_{+}, \beta_{+}\right)$. Then we have by (3.1) that

$$
0=\left|\left\langle L v-g(v), P_{2} v\right\rangle\right|=\left|F^{\prime}\left(\left\|P_{2} v\right\|\right)\right|>0 .
$$

This is a contradiction. Therefore we have that $\left\|P_{2} v\right\| \in\left(\alpha_{+}, \beta_{+}\right)$and $v \in U_{+}$. Then from (2.12) and (2.16), we find that

$$
\|L w-g(w)\|_{*}\|w-v\| \geq\langle L w-g(w), w-v\rangle \geq \omega_{2}\|w-v\|^{2}
$$

for all $w \in V$ with $P_{2} w=P_{2} v$. Noting that $P_{2} v_{n} \rightarrow P_{2} v$, we see that

$$
\lim \left\|L w_{n}-g\left(w_{n}\right)\right\|_{*}=\lim \left\|L v_{n}-g\left(v_{n}\right)\right\|_{*}=0,
$$

where $w_{n}=v_{n}-P_{2} v_{n}+P_{2} v$ for each $n \geq 1$. Then combining (3.4) with (3.3), we obtain that $v_{n}$ converges to $v$ strongly in $V$. Since $K_{+} \backslash U_{+}$is strongly closed in $V$, we find that $v \in K_{+} \backslash U_{+}$. This is a contradiction. Thus we have shown that there exists $d_{+}>0$ such that (3.2) holds for all $v \in K_{+} \backslash U_{+}$. By the same argument, we have that there exists $d_{-}>0$ satisfying (3.2) for all $v \in K_{-} \backslash U_{-}$.

Hence we consider the initial value problem of the form

$$
\begin{gathered}
\frac{d u}{d t}+L u=g(u)+h, \quad 0<t<T, \\
u(0)=u_{0},
\end{gathered}
$$


where $u_{0} \in H$. Let $h \in W^{1,1}(0, T ; H)$ and $u_{0} \in H$. Then since $g$ is Lipschitz continuous, the problem (3.5) has a unique solution $u \in W^{1, \infty}(0, T ; H)$ such that $u(t) \in V$, a.e. $(0, \infty)$. (See, for example, $[4,5$ or 6$]$.) Noting that $d u / d t \in$ $L^{\infty}(0, T ; H)$ and

$$
\Phi(u(t))-\Phi(u(s))=\int_{s}^{t}\left\langle\Phi^{\prime}(u(\tau)), u^{\prime}(\tau)\right\rangle d \tau=\int_{s}^{t}\left\langle h(\tau)-\frac{d u}{d t}, \frac{d u}{d t}\right\rangle d \tau
$$

for $0<s \leq t<T$, we can see that the mapping $t \rightarrow \Phi(u(t))$ is continuous.

For each $h \in W^{1, \infty}(0, T ; H)$ and $w \in H$, we set $T_{h} w=u(T)$, where $u$ is the solution of (3.5) with $u_{0}=w$. Then the mapping $T_{h}: H \rightarrow H$ is continuous. (See also [6].)

Lemma 6. There exists $M>0$ such that $T_{h} w \in K_{ \pm}$, for all $w \in K_{ \pm}$and all $h \in W^{1, \infty}(0, T ; H)$ with $\sup \{|h(t)|: t \in(0, t)\} \leq M$.

Proof. By Lemma 5 , it is easy to verify that there exist a positive number $M>0$ and a negative number $c_{*}>c$ such that

$$
\|L v-g(v)\|_{*} \geq M \quad \text { for all } v \in W_{ \pm} .
$$

where $W_{ \pm}=\left\{v \in V: \Phi(v)<c_{*}, P_{2} v \in\left(\alpha_{ \pm}^{*}, \beta_{ \pm}^{*}\right)\right\} \backslash U_{ \pm}$. Let $w \in K_{+}$and $h \in W^{1, \infty}(0, T ; H)$ such that $\sup \{|H(t)|: t \in(0, T)\} \leq M$. Let $u:(0, T) \rightarrow$ $V$ be the solution of the problem (3.5) with $u_{0}=w$. To prove Lemma 6, it is sufficient to show that $\Phi(u(t)) \leq c$ for all $t \in(0, T)$. Suppose that $\Phi(u(t)) \leq c$ on $(0, s)$ and $\Phi(u(s))=c$ for some $0 \leq s<T$. Then we have that $u(s) \in K_{+} \backslash U_{+}$. Since the mapping $t \rightarrow \Phi(u(t))$ is continuous, we find that there exists $s^{*} \in(s, T)$ such that $u(t) \in W_{+}$on $\left(s, s^{*}\right)$. Then from (3.6), we find that

$$
\begin{aligned}
\Phi(u(t))-\Phi(u(s)) \\
\quad=\int_{s}^{t}\left\langle\Phi^{\prime}(u(\tau)), u^{\prime}(\tau)\right\rangle d \tau \\
=\int_{s}^{t}\langle L u(\tau)=g(u(\tau)),-(L u(\tau)-g(u(\tau)))+h(\tau)\rangle d \tau \\
\quad \leq \int_{s}^{t}\left(-|L u(\tau)-g(u(\tau))|^{2}+|L u(\tau)-g(u(\tau))||h(\tau)|\right) d \tau \\
\quad \leq \int_{s}^{t}\left(-\|L u(\tau)-g(u(\tau))\|_{*}^{2}+\|L u(\tau)=g(u(\tau))\|_{*}|h(\tau)|\right) d \tau \\
\leq-M^{2}(t-s)+M \cdot \sup \{|h(t)|: t \in(0, T)\}(t-s) \leq 0
\end{aligned}
$$

for all $t \in\left(s, s^{*}\right)$. Therefore $\Phi(u(t)) \leq c$ for all $t \in\left(s, s^{*}\right)$. Thus we have shown that $\Phi(u(t)) \leq c$ on $(0, T)$. This completes the proof.

We can now complete

Proof of Theorem. Let $h \in W^{1, \infty}(0, T ; H)$ such that $\sup \{|h(t)|: t \in(0, T\} \leq$ $M$. From the definition of $T_{h}$, the fixed points of $T_{h}$ are the periodic 
solutions of $(\mathrm{P})$. Here we consider the mapping $E T_{h} Q_{+}: \widetilde{K}_{+} \rightarrow \widetilde{K}_{+}$. From the observation above, $T_{h} Q_{+}$is continuous with respect to the norm of $H$. Then since $\widetilde{K}_{+}$is compact and convex in $H, T_{h} Q_{+}$has a fixed point $w_{+} \in \widetilde{K}_{+}$. Noting that $Q_{+} w_{+} \in K_{+} \subset\{v \in V: \Phi(v) \leq c\}$, we have by Lemma 6 that $w_{+}=T_{h} Q_{+} w_{+} \in K_{+}$. Then $w_{+}=Q_{+} w_{+}$and $w_{+}=T_{h} w_{+}$. Therefore the solution $u_{+}$of the problem (3.5) with the initial value $u_{0}=w_{+}$is a $T$-periodic solution. By the same argument, we have that there exists a solution $u_{-}$of $(\mathrm{P})$ satisfying $u_{-}(0) \in K_{-}$. Then since $K_{+} \cap K_{-}=\{\phi\}$, the problem $(\mathbf{P})$ has at least two solutions.

\section{REFERENCES}

1. S. Ahmad, Multiple nontrivial solutions of resonant and nonresonant asymptotically linear problems, Proc. Amer. Math. Soc. 96 (1986), 405-409.

2. V. Amann, Saddle points and multiple solutions of differential equations, Math. Z. 169 (1979), 127-166.

3. __ Periodic solutions for semilinear parabolic equations, Nonlinear Analysis, Academic Press, New York, N.Y., 1978, pp. 1-29.

4. V. Barbu, Nonlinear semigroups and evolution equations in Banach spaces, Noordhoff, Leyden, 1976.

5. F. E. Browder, Non-linear equations of evolution, Ann. of Math. (2) 80 (1964), 485-523.

6. M. G. Crandall and A. Pazy, Nonlinar evolution equations in Banacah spaces, Israel J. Math. 11 (1972), 57-94.

7. N. Hirano, Multiple nontrival solutions of semilinear elliptic equations, Proc. Amer. Math. Soc. 103 (1988), 468-472.

8. J. J. Nieto, Periodic solutions of nonlinear parabolic equations, J. Differential Equations 60 (1985), 90-102.

Department of Mathematics, Faculty of Engineering, Yokohama National UniverSITY, YOKOHAMA, JAPAN 